
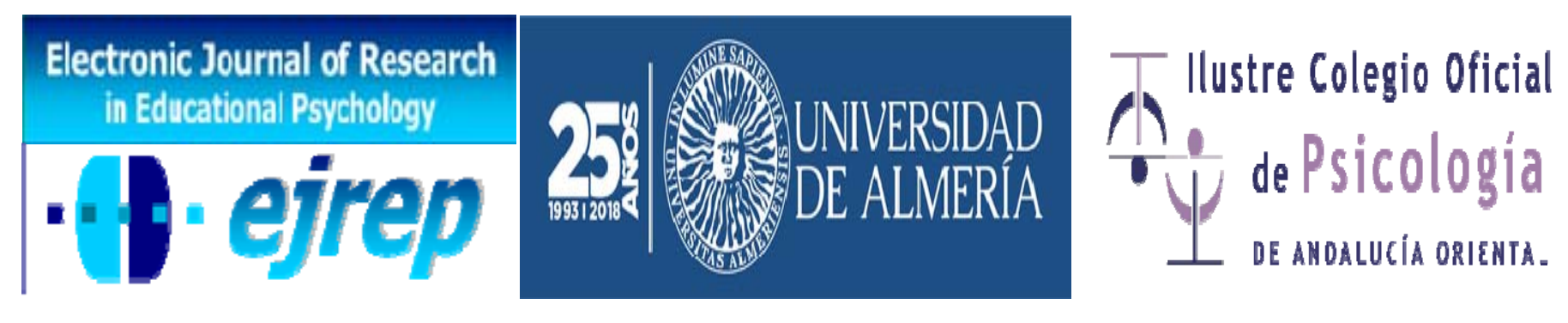

de andalucía orienta.

\title{
Atención e impulsividad en Niños con Alta Capacidad Intelectual y Niños con TDAH
}

\section{María de los Dolores Valadez Sierra ${ }^{1}$, Luis Alexis Aguiña- ga Maldonado ${ }^{2}$, José Salvador Morales González ${ }^{2}$, Emilio Verche Borges ${ }^{3}$, África Borges del Rosal ${ }^{4}$ y Celia Josefina Rodríguez Cervantes ${ }^{5}$}

${ }^{1}$ Departamento de Psicología Aplicada, Centro Universitario Ciencias de la Salud, Universidad de Guadalajara, México

${ }^{2}$ Licenciatura en Psicología, Centro Universitario de Ciencias de la Salud, Universidad de Guadalajara, México

${ }^{3}$ IE University, Madrid, España

${ }^{4}$ Facultad de Ciencias de la Salud, Universidad de La Laguna

${ }^{5}$ Doctorado Interinstitucional en Psicología, Centro Universitario Ciencias de la Salud, Universidad de Guadalajara, México

México /España

Correspondencia: María de los Dolores Valadez Sierra. Isla Pantenaria 2375, Jardines de San José, Guadalajara, Jalisco, México. E-mail: doloresvaladez@yahoo.com.mx

(C) Universidad de Almería and Ilustre Colegio Oficial de la Psicología de Andalucía Oriental (Spain) 


\section{Resumen}

Introducción. Comportamientos asociados a la alta capacidad intelectual, como la inatención y respuesta anticipada amenudo se confunden con el trastorno por déficit de atención e hiperactividad (TDAH) y dificultan su identificación. El objetivo del presenta trabajo fue analizar el desempeño de los alumnos con alta capacidad intelectual y alumnos con TDAH en la atención sostenida y la impulsividad.

Método. Participaron un total de 25 niños con alta capacidad intelectual, edad promedio 6.15 años y 25 niños con TDAH, edad promedio de 6.96 años, evaluados mediante el Conner's Continuous Performance Test II for Windows (CPT II). Se ha seguido una metodología ex post facto. Los resultados se analizaron estadísticamente, utilizando el programa SPSS, mediante una prueba t para muestras independientes.

Resultados. Se encontraron diferencias significativas en Omisiones, Tiempo de Reacción, Error del Tiempo de Reacción y Tiempo de Reacción a Estímulo al Cambio de Intervalo Entre Estímulo.

Discusión y conclusiones. (1) Los niños con alta capacidad intelectual poseen significativamente una mejor consistencia en la velocidad de respuesta, mejor consistencia en los tiempos de reacción en los distintos intervalos inter-estímulo y cometen mayores errores de comisión en comparación con los alumnos con TDAH. (2) Los niños con alta capacidad intelectual ejecutan significativamente más rápido al responder correctamente durante la prueba. Las pruebas de rendimiento como el CPT-II para la medición de la atención y la impulsividad representan una alternativa de evaluación, de diagnóstico diferencial entre estas poblaciones; este proceso debe ser realizado por personal calificado y con experiencia con ambas poblaciones.

Palabras Clave: inatención, impulsividad, altas capacidades, tdah. 


\begin{abstract}
Introduction. Behaviors associated with high intellectual capacity, such as inattention and early response are confused with attention deficit hyperactivity disorder (ADHD) and make it difficult to identify. The objective: to analyze the performance of students with high intellectual capacity and students with ADHD in sustained attention and impulsivity.
\end{abstract}

Method. A total of 25 children with high intellectual capacity, mean age 6.15 years and 25 children with ADHD, mean age of 6.96 years, were evaluated by Conner's Continuous Performance Test II for Windows (CPT II). A quantitative, descriptive, cross-sectional, nonexperimental study were utilized. The results were statistically analyzed using SPSS: t-test for independent samples.

Results. There were significant differences in the follow inattention variables: Omissions, Hit Reaction Time and Hit Standard Error Inter Stimulus Interval Change; and in the variable of impulsivity: Hit Reaction Time.

Discussion and Conclusion. (1) Children with high intellectual ability have a significantly better consistency in response speed, better consistency in reaction times at different interstimulus intervals, and make higher commission errors compared to students with ADHD; (2) children with high intellectual ability perform significantly faster and correctly during the test. Performance tests such as the CPT-II for the measurement of attention and impulsivity represent an alternative assessment, of differential diagnosis among these populations. This process must be carried out by qualified and experienced professional in both populations.

Keywords: inattention, impulsivity, intellectual ability, adhd 


\section{Introducción}

Si bien la alta capacidad intelectual y el trastorno de deficit de atención con hiperactividad no son diagnósticos excluyentes, pudiendo en ciertos casos coexistir, lo cierto es que con frecuencia se observa que tanto los docentes como los profesionales de la educación se les dificulta distinguir entre niños con trastorno de déficit de atención con hiperactividad y con alta capacidad intelectual (Orendorff, 2009). Existen comportamientos asociados a la alta capacidad intelectual, tales como la falta de atención (en aquellas actividades que le aburren o no son de su interés), o el responder anticipadamente (por conocer la respuesta), entre otros, que también pueden ser comportamientos característicos del TDAH (Webb y Latimer, 1993; Hernández y Borges 2005, Valadez, 2012).

Webb y Latimer (1993) señalaban que frecuentemente, los niños con alta capacidad intelectual eran mal diagnosticados por mostrar ciertas conductas, tales como inquietud, inatención, impulsividad, soñar despierto, entre otras, por lo que recibian erroneamente el diagnóstico de Trastorno de Deficit de la Atención e Hiperactividad (TDAH). Por su parte Hernández y Borges (2005) así como Antshel (2008), refieren que la desatención puede ocurrir en los alumnos con alta capacidad intelectual en la medida en que no estan siendo desafiados y se encuentran en un ambiente educativo aburrido, con tareas repetitivas y carentes de sentido, ocasionando en la mayoría de las veces que estos niños pierdan hasta más del $50 \%$ de su tiempo esperando a que el resto de los compañeros comprendan las tareas, lo que puede conducir a que demanden atención, presenten conductas disruptivas en el aula, se se nieguen a realizar las tareas o bien a no apuntar los deberes que se les manda (Sanz, 2017). Por ello, no es de extrañar que que muchas características de los alumnos con alta capacidad intelectual se confundan con los síntomas de TDAH (Mullet \& Rinn, 2015).

De acuerdo al DSM V (Asociación Americana de Psiquiatría, 2013), el TDAH es un Trastorno del neurodesarrollo que se caracteriza por un patrón persistente de inatención y/o hiperactividad-impulsividad que interfiere con el funcionamiento o desarrollo del niño. Los comportamientos que puede presentar son que a menudo no presta suficiente atención a los detalles, no mantiene la atención en las actividades, parece no escuchar cuando se le habla, no sigue instrucciones y no finaliza tareas, se mueve o habla en exceso, o emite respuestas precipitadas antes de que haya finalizado la pregunta, entre otros. 
En contraste, los niños con alta capacidad intelectual pueden presentar comportamientos como: pobre atención, especialmente en aquellas actividades que han dominado rapidamente, aburrimiento y soñar despiertos en situaciones especificas, alta excitabilidad imaginativa, baja toletancia a persistir en tareas que les parecen irrelevantes, resistencia a practicar habilidades que aún no han dominado, el desarrollo del juicio va retrasado en comparación al desarrollo general de la inteligencia, posibles problemas de poder con la autoridad, altos niveles de actividad llegando incluso a necesitar menos horas de sueño, cuestionar las reglas, costumbres y tradiciones (Neihart \& Betts, 2010).

Muchos de los diagnosticos de TDAH que se realizan se hacen en base a criterios que enlistan comportamientos. El problema con esta forma de diagnosticar es que solo toma en cuenta los comportamientos observables del menor, en lugar de las causas de dichos comportamientos en cada individuo (Orendorff, 2009). Resulta necesario analizar en cada niño la situación y el origen del comportamiento para poder determinar cuando un niño tiene TDAH o alta capacidad, ya que los orígenes de ambas condiciones difieren considerablemente. Respecto al TDAH, la etiología puede tratarse de una deficiencia cognitiva, mientras que en el caso de los menores con alta capacidad intelectual sus comportamientos pueden deberse a falta de motivación, aburrimiento y sobre-excitabilidad (Hartnett, Nelson, \& Rinn, 2003; Benito, 2001).

Según Benito (2001), la conducta intensa y curiosa de los niños con alta capacidad, exacerbada por el aburrimiento en clase, se asemeja a la conducta de los niños con TDAH, confusión que afecta principalmente a los profesores y padres de los menores. Maureen (2003), considera que resulta difícil distinguir entre verdaderos deficits de atención y los comportamientos y temperamentos tipicos en niños con alta capacidad intelectual, existiendo por esto una gran preocupación entre los profesionales de la salud mental por los posibles errores diagnosticos al patologizar la conducta normal de las personas con altas capacidad. En ambas poblaciones aparece interés por termas específicos, dificultades en el seguimiento de las clases y sus contenidos, asi como un déficit para relacionarse con sus pares de la misma edad (Benito, 2001).

Son pocos los estudios empiricos que brinden datos concretos o una aproximación certera acerca de cuan frecuentemente los niños o adultos con alta capacidad intelectual estan siendo mal diagnosticados con otros trastornos mentales, entre ellos el TDAH, la mayoría de los investigadores especialistas en el tema concuerdan en que el mal diagnostico es un 
fenomeno que sucede y posee altas probabilidades de sucitarse, especialmente en relación con el Trastorno por Deficit de Atención/Hiperactividad debido a la similitud de su sintomatología (Lovecky, 2014; Rinn \& Reynolds, 2012; Webb, Goerss, Amend, Webb, Beljan \& Olenchak, 2006).

En la practica clinica, refieren los autores, han atendido a niños cuyos padres han acudido a ellos por una segunda o tercera opinion, y que en su experiencia clínica pareciera que los comportamientos tipicos de los niños superdotados podrian estan siendo confundidos con trastornos del comportamiento en más del $25 \%$ de los casos. Entre los trastornos que mayor mal diagnostico causan en los niños superdotados son: TDAH, Trastorno de Asperger, Trastorno Obsesivo Compulsivo, Trastorno Oposicionista Desafiante y Trastorno Bipolar. Así pues un diagnostico oportuno sería aquel proveniente de un equipo de profesionales expertos en el tema (Webb, Goerss, Amend, Webb, Beljan \& Olenchak, 2006).

Por lo tanto, el mal diagnostico de un niño con alta capacidad o con TDAH puede suceder debido las similtudes entre poblaciones, las peculiaridades de cada individuo o las numerosas dificultades en el proceso de identificacion, entre las cuales se encuentra el desconocimiento de los profesionales de la educación y personal que realiza el diagnóstico respecto a una o ambas condiciones, además de un posible ambiente de aprendizaje inapropiado (Edwards, 2009). Y resulta esto de especial relevancia, debido al impacto negativo que tiene en el individuo recibir un diagnostico incorrecto, volviendose sumamente improbable que sus necesidades sean correctamente satisfechas (Fisher, 2013).

Uno de los instrumentos de amplio uso para el diagnóstico del TDAH es el CPT II (Conners y MHS, 2013), que se ha utilizado tanto para el diagnóstico de niños con TDAH como en estudios comparativos entre niños con y sin TDAH. De la misma forma, se ha utilizado en población con y sin alta capacidad, como en el estudio de Shi et al (2013), en el cual concluyen que el rendimiento de estos es superior en comparación al de sus pares con inteligencia promedio; especificamente, tienen mejor capacidad de concentrar su atención, mejor contención de impulsos ante tareas de cotidianidad, demostrando una mejor precaución y acción ante dichas actividades, así como una mejor sensibilidad a los estimulos de la prueba. Sin embargo, gran parte de la muestra de los alumnos con alta capacidad ya contaba con tres o más años de enriquecimiento lo que puede ser una variable que puede haber influido en los resultados. De igual manera Benito y Guerra (2014), compararon los resultado en la prueba CPT II de niños con alta capacidad intelectual con y sin TDAH, y de niños con 
TDAH con y sin alta capacidad. Este estudio constituye una aproximación al abordaje de ambas poblaciones, aunque, sin embargo, se observa que el rango de edad de la población estudiada fue muy grande (de 4 a 20 años, dándose el caso de que en algunas de las funciones ejecutivas, como es la atención, se encuentran picos en el desarrollo) y se observa en los resultados que no se realizó una comparación entre alumnos con alta capacidad intelectual y alumnos con TDAH.

\section{Objetivos e hipótesis}

El objetivo del presente estudio fue analizar el rendimiento entre niños con Alta Capacidad Intelectual y niños con TDAH en la atención sostenida y la impulsividad evaluada a traves de la prueba de CPT II.

\section{Método}

\section{Participantes}

Participaron 50 niños que asistieron a evaluación al Laboratorio de Psicología y Educación Especial del Departamento de Psicología Aplicada del Centro Universitario de Ciencias de la Salud de la Universidad de Guadalajara (Méjico), quienes, en base a su diagnóstico, fueron distribuidos en dos grupos: el primer grupo, de alta capacidad intelectual (Coficiente Intelectual superior a 125 , obtenido mediante las escalas de Inteligencia Wechsler) formado por 25 niños (de los cuales 17 eran varones, cuya edad promedio fue de 6.15 años y con una desviación estándar de 1.15 años. El segundo grupo de TDAH, se conformó por una niña y 24 niños, diagnosticados con el trastorno bajo los criterios del DSM-V. La edad promedio de este grupo fue de 6.96 años y una desviación estándar de 0.97 años.

\section{Instrumentos}

Conner's Continuous Performance Test II for Windows (CPT II) (Conners y MHS, 2013). Para la aplicación se requiere que el programa esté instalado en una computadora con sistema operativo Windows. Se le pide a la persona evaluada que presione la barra espaciadora en el teclado de la computadora o haga click con el botón izquierdo del ratón cada vez que aparezca una letra en la pantalla, excepto cuando aparezca la letra "X". Los estímulos se organizan en seis bloques y estos a su vez en tres sub-bloques con 20 estímulos cada uno, en los 
que entre cada estímulo puede haber lapsos de 1, 2 y 4 segundos con un tiempo de exhibición de 250 milisegundos. El tiempo total de aplicación es de 14 minutos.

Al terminar la aplicación el programa genera los resultados automáticamente en un grupo de tablas con datos crudos y análisis estadísticos de los mismos. El programa captura los tiempos de respuesta midiendo velocidad y consistencia. Las respuestas con un tiempo de reacción inferior a los $100 \mathrm{~ms}$ se consideran como perseveraciones, debido a que es virtualmente imposible reaccionar tan rápidamente. Las variables analizadas se agrupan en dos bloques: inatención e impulsividad. En la tabla 1 se presentan las distintas variables analizadas y a qué bloque se corresponden.

Tabla 1. Variables analizadas del CPT

\begin{tabular}{lccc}
\hline Escala & Inatención & Impulsividad & Errores \\
\hline Omisiones & $\mathrm{X}$ & & $\mathrm{X}$ \\
Comisiones & $\mathrm{X}$ & $\mathrm{X}$ & $\mathrm{X}$ \\
Tiempo de reacción al estímulo & $\mathrm{X}$ & $\mathrm{X}$ & \\
Error típico de TRE & $\mathrm{X}$ & $\mathrm{X}$ & \\
Variabilidad & $\mathrm{X}$ & $\mathrm{X}$ & \\
Detectibilidad & $\mathrm{X}$ & $\mathrm{X}$ &
\end{tabular}

Estilo de respuesta

$\begin{array}{ll}\text { Perseveraciones } & \mathrm{X}\end{array}$

TRECB

Error típico de TRECB

TRECIEE

$\mathrm{X}$

Error típico de TRECIEE $\quad \mathrm{X}$

Nota: TRE: Tiempo de reacción al estímulo; TRECB: Tiempo de Reacción a Estímulo por Cambio de Bloque; TRECIEE: Intervalo Entre Estimulo. 


\section{Procedimiento}

Se aplicó la prueba computarizada Continuous Performance Test II (CPT II) V.5 para Windows a los niños diagnosticados con TDAH y a niños identificados con alta capacidad intelectual, en el Laboratorio en Psicología y Educación Especial (LAPSIEE) del Centro Universitario de Ciencias de la Salud de la Universidad de Guadalajara. Todas las aplicaciones se realizaron en una misma computadora y los aplicadores fueron capacitados específicamente para esta tarea.

\section{Análisis de datos}

Con objeto de comprobar si existen diferencias en las puntuaciones obtenidas entre el grupo de altas capacidades frente al de TDAH se realizó un contraste de t de Student para muestras independientes, mediante el programa SPSS (Versión 21 para Windows), calculándose para determinar el tamaño del efecto la g de Hedges.

\section{Resultados}

Se obtuvieron las medias y desviación estándar de todas las variables del CPT II por cada uno de los grupos. Los resultados se muestran en la tabla 2. 
Tabla 2. Medias y Desviación de las puntuaciones de cada una de las variables del CPT II obtenidas por cada grupo

\begin{tabular}{llllll}
\hline Población & AACC & TDAH & $\mathrm{t}(48)$ & $\mathrm{P}<$ & $\mathrm{g}$ \\
\hline Omisiones & $55.75(12.16)$ & $73.79(20.73)$ & -3.75 & 0.001 & $-1,04$ \\
Comisiones & $50.63(8.84)$ & $52.10(5.72)$ & -0.71 & 0.438 & $-0,19$ \\
TRE & $52.63(9.14)$ & $67.60(12.74)$ & -4.77 & 0,001 & $-1,33$ \\
Error TRE & $53.27(7.83)$ & $65.86(11.07)$ & $-4,74$ & $<0.001$ & $-1,29$ \\
Variabilidad & $53.02(9.33)$ & $58.19(14.21)$ & -1.52 & 0.135 & $-0,66$ \\
Detectabilidad & $52.24(7.88)$ & $60.22(25.00)$ & -1.52 & 0.135 & $-0,50$ \\
Estilo de respuesta & $54,68(21,34)$ & $62,16(19,13)$ & -1.31 & 0.198 & -0.36 \\
Perseveraciones & $50.42(12.89)$ & $53.46(26.04)$ & -0.52 & 0.604 & $-0,15$ \\
TRECB & $50.13(10.59)$ & $55.54(11.30)$ & -1.74 & 0.087 & $-0,49$ \\
Error típico TRECB & $50.72(9.12)$ & $51.15(8.91)$ & -0.167 & 0.868 & $-0,05$ \\
TRECIEE & $55.84(8.98)$ & $62.42(14.75)$ & -1.96 & 0.063 & $-0,53$ \\
Error típico TRECIEE & $51.58(8.43)$ & $57.35(8.32)$ & -2.44 & 0.019 & $-0,68$ \\
\hline
\end{tabular}

Nota: media (desviación típica); TRE: Tiempo de Reacción al Estímulo; TRECB: Tiempo de Reacción a Estímulo por Cambio de Bloque; TRECIEE Tiempo de Reacción a Estímulo al Cambio de Intervalo entre Estímulo (TRECIEE)

Como se puede observar en la tabla 2, la t de students indicó diferencias estadísticamente significativas en las variables de Omisiones, Tiempo de Reacción, Error del Tiempo de Reacción y Tiempo de Reacción a Estímulo al Cambio de Intervalo Entre Estimulo. Los niños con alta capacidad intelectual cometieron menos omisiones, presentaron significativamente mejor consistencia en la velocidad de respuesta, mejor consistencia en los tiempos de reacción en los distintos intervalos inter-estímulo y cometieron mayores errores de comisión en comparación con los alumnos con TDAH 


\section{Discusión y Conclusiones}

El objetivo del presente estudio consistió en en analizar el rendimiento de los niños con alta capacidad intelectual en comparación con el de niños con TDAH y conocer las similitudes y diferencias entre ambas poblaciones. Los resultados del presente estudio indican que entre los niños con TDAH y los niños con alta capacidad intelectual existen diferencias significativas en el rendimiento atencional y la conducta impulsiva, teniendo los niños con alta capacidad intelectual mejores indicadores atencionales (tiempo de respuesta y omisiones) y menor impulsividad.

Se encontró que los niños con TDAH realizaron más omisiones, puntuaciones que resultaron significativamente más elevadas con respecto a los niños con alta capacidad intelectual. Este hallazgo es similar al de otros estudios que incluyeron sujetos de edades variadas, en el que los sujetos con TDAH cometen más omisiones y comisiones, pero, no obstante, hay pocas diferencias significativas en las demás medidas del CPT ( Epstein, Conners, Sitarenios, \& Erhardt, 1998).

De la misma manera, el hallazgo concuerda con los resultados del estudio de Benito y Guerra (2014), en el que se comparó el rendimiento en la prueba de niños superdotados con y sin TDAH y niños con TDAH con y sin superdotación intelectual, encontrando igualmente que los niños con TDAH con y sin superdotación intelectual cometieron más errores de omisión que las otras poblaciones. Asimismo, los autores concluyen que los niños con TDAH sin superdotación intelectual obtuvieron puntuaciones más elevadas que los superdotados con TDAH en esta medida. Los resultados aquí obtenidos concuerdan con los de Rosengren (2004), quien comparó el desempeño en CPT de niños superdotados y niños con inteligencia normal y encontró que los superdotados presentaban un mejor rendimiento en la prueba, expresado en un tiempo de respuesta más rápido, menor variabilidad en la respuesta a lo largo de la prueba y una mejor atención a los estímulos que los niños con inteligencia normal.

También, Shi y otros (2012) en su estudio comparativo argumentan que en su muestra los niños con alta capacidad intelectual cometen significativamente menos errores de omisión, menos errores de comisión y poseen una menor habilidad para distinguir entre estímulos objetivo y no objetivo (variable Detectabilidad d') en comparación a niños con inteligencia normal. En nuestro estudio apoyamos solo parcialmente los resultados expuestos por Shi y otros 
(2012) ya que concordamos en que los menores con alta capacidad intelectual cometen significativamente menos errores de omisión, pero no encontramos diferencias significativas en los errores de comisión, muy al contrario, los resultados de la variable comisiones resultan ser muy similares en nuestras muestras, del mismo modo no encontramos diferencias entre poblaciones respecto a la habilidad para distinguir entre estímulos objetivo y estímulos noobjetivo mediante la medida Detectabilidad d'. No obstante, sí se ha obtenido en la presente investigación que los tiempos de reacción difieren significativamente entre los grupos, situación que no sucede en el estudio de Shi y otros (2012), probablemente porque su grupo comparativo se conformaba de niños con inteligencia normal sin ningún trastorno psiquiátrico asociado, a diferencia de la presente muestra, con menores diagnosticados con TDAH.

Es necesario destacar que, si bien existen diferencias significativas entre poblaciones en algunas medidas tanto de inatención como de impulsividad, la mayoría de las medidas evaluadas con el CPT II comparadas no resultaron significativa. Del mismo modo, es importante hacer notar que desde un análisis cualitativo la medida de comisiones, perteneciente tanto a inatención como a impulsividad, mostró resultados muy similares en ambas poblaciones, lo que nos indica que los niños con alta capacidad intelectual y los niños con TDAH en nuestra muestra cometen un nivel similar de errores de comisión.

El mal diagnostico entre niños con TDAH y alta capacidad intelectual es un fenómeno que afecta la correcta detección e intervención de los menores (Webb, Goerss, Amend, Webb, Beljan \& Olenchak, 2006). Las pruebas de rendimiento como el CPT II para la medición de la atención y la impulsividad se presentan como una alternativa de evaluación que pueden contribuir a un mejor diagnostico diferencial entre estas poblaciones (Moreno García, Delgado Pardo, Aires González, \& Meneres Sancho, 2013).

Finalmente, los resultados aquí obtenidos permiten concluir que el proceso de evaluación y diagnostico de niños con trastorno por déficit de atención/hiperactividad, así como el proceso de identificación de niños con alta capacidad intelecual debe ser realizado por personal calificado y con experiencia en ambas poblaciones, con el objetivo de evitar la confusión entre poblaciones que puede llevar a un mal diagnostico. Así mismo, la interpretación y utilización de la prueba CPT II durante el proceso diagnóstico diferencial debe realizarse con cautela y solo por profesionales expertos en ella. Así mismo, derivado de este estudio, se recomienda incorporar en la batería para el diagnóstico la evaluación de funciones ejecutivas. 
Una de las limitaciones del presente estudio fue el tamaño de la muestra, por lo que no se puede realizar una generalización de los resultados, no obtante aportó información útil en la comprensión del funcionamiento en estas dos variables en ambas poblaciones.

\section{Referencias}

Antshel, K. M. (2008) Attention-Deficit Hyperactivity Disorder in the context of a high intellectual quotient/giftedness. Developmental Disabilities Research Reviews, 14, 293299. https://doi.org/ 10.1002/ddrr.34.

Asociación Americana de Psiquiatría. (2013). Manual Diagnóstico y Estadístico de los Trastornos Mentales ( $5^{a}$ ed.). Arlington, V. A.: American Psychiatric Publishing

Benito, Y. (2001). ¿Existen los superdotados?.España: CissPraxis.

Benito, Y. y Guerra, S. (2014). Diagnostico del Trastorno por Deficit de Atención con Hiperactividad (TDAH) en niños con Superdotación Intelectual. Universidad de Burgos.

Conners, K., y MHS Staff. (2013). Conners Continuous Performance Test for Windows CPT II. Toronto: MHS.

Edwards, K. (2009). Misdiagnosis, the Recent Trend in Thinking about Gifted Children with ADHD. APEX, 29-44.

Epstein, J., Erkanli, A., Conners, K., Klaric, J., Costello, J. y Angold, A. (2003). Relations Between Continuous Performance Test Performance Measures and ADHD Behaviors. Journal of Abnormal Child Psychology, 31(5), 543-554. https://doi.org/10.1023/A:1025405216339

Fisher, T. (04 de 09 de 2013). Medical Misdiagnosis in the Gifted. Obtenido de Education Week Teacher: http://blogs.edweek.org/teachers/unwrapping_the_gifted/2013/09/medical_misdiagnos is_in_the_gi.html

Hartnett, D. N., Nelson, J. M., \& Rinn, A. N. (2003). Gifted or ADHD? The Possibilities of $\begin{array}{lllll}\text { Misdiagnosis. } & \text { Roeper } & \text { Review } & \text { 73-76(2), }\end{array}$ https://doi.org/10.1080/02783190409554245

Hernández, C. y Borges, A. (2005). Un programa de aprendizaje autorregulado para personas de altas capacidades mediante el uso de herramientas telemáticas. Electronic Journal of Research in Educational Psychology 3(3), 233-252. 
Lovecky, D. V. (2014). Misperceptions about giftedness and diagnosis of ADHD and other disorders. Providence, RI: Gifted Resource Center of New England.

Maureen, N. (2003). Gifted Children with Attention Deficit Hyperactivity. ERIC Clearinghouse on Disabilities and Gifted Education. Obtenido el 25 de febrero de 2018 de ERIC database: Education Resource Information Center.

Moreno García, I., Delgado Pardo, G., Aires González, M. y Meneres Sancho, S. (2013). Aplicación del CPT/IVA en la valoración de los efectos del Neurofeedback en el TDAH. Anuario de Psicología Clínica y de la Salud, 9, 57-59.

Mullet, D. y Rinn, A. (2015). Giftedness and ADHD: Identification, Misdiagnosis, and Dual $\begin{array}{llll}\text { Diagnosis. } & \text { Roeper } & \text { Review, } & \text { 37(4), }\end{array}$ https://doi.org/10.1080/02783193.2015.1077910

Neihart, M. y Betts, G. (2010). Revised Profiles of the Gifted \& Talented. Recuperado de Talent Stimuleren: http://talentstimuleren.nl/?file=553\&m=1370389145\&action=file.download

Orendorff, K. A. (2009). The Relationship Between ADHD and Giftedness. San Rafael, CA: Dominican University of California.

Rinn , A. N. y Reynolds, M. J. (2012). Overexcitabilities and ADHD in the Gifted: An Examination. Roeper Review, 38-45. http://dx.doi.org/10.1080/02783193.2012.627551

Rosengren, K. S. (2004). Performance of Intellectually Gifted Children on Three Measures of Attention Deficit Hyperactivity Disorder. George Fox University, 1(1).

Shi J, Tao T, Chen W, Cheng L, Wang L, et al. (2013). Sustained Attention in Intellectually Gifted Children Assessed Using a Continuous Performance Test. PLOS ONE 8(2): e57417. doi:10.1371/journal.pone.0057417

Valadez, M. D. (2012). Identificación y Evaluación de Alumnos Superdotados y Talentosos. En: M. D. Valadez, J.Betancourt y A, Zavala. Alumnos superdotados y talentosos. México: Manual Moderno

Webb, J. T. y Latimer, D. (1993). ADHD and children who are gifted. Exceptional Children 60, 183-185.

Webb, J. T., Meckstroth, E. A. y Tolan, S. S. (1994). Guiding the Gifted Child. Scottsdale, Arizona: Gifted Psychology Press.

Webb, J., Goerss, J., Amend, E., Webb, N., Beljan, P. y Olenchak, F. (2006). Diagnosis or Misdiagnosis. Understanding Our Gifted, 18(2), 15-17. 